\title{
On ways of knowing in medicine
}

$\mathrm{E}$ vidence-based medicine, translational medicine, narrative medicine, personalized medicine and precision medicine are all specially coined terms that have been used to herald new ways of knowing in medicine. I have listed the terms in chronological order, with evidencebased medicine starting around 1992 and precision medicine around 2011, making five methodologies in just one generation. I have been curious about what these terms mean, and what their roles are - substantive and rhetorical — in research and clinical contexts.

The most useful question to ask is "What lacuna is this new kind of medical knowledge intended to fill?" It turns out that each fills an important perceived need, and we can understand the new terms better by making these needs explicit and seeing how they unfold over time.

Evidence-based medicine (first mentioned in the literature in 1992) ${ }^{1}$ developed after the expansion of clinical trials in the post-World War II era. Both the number and quality of clinical trials were increased in the second half of the 20th century and, typically, new interventions were tested in multiple trials. Although randomized controlled trials (RCTs) were valued as providing the highest quality evidence, there was no explicit process for assessment of overall evidence - varying in quality and from different trials - for or against a medical intervention. In fact, this was standardly left to a consensus of experts to decide, based on their familiarity with all the relevant clinical trials. ${ }^{2,3}$ When the evidence was clear and univocal, a consensus of experts was easily accepted. However, if the evidence was equivocal or discordant (i.e., different trials had contrary results), the objectivity of expert consensus was easily challenged. Evidence-based medicine provides explicit and (it is claimed) objective procedures for assembling and evaluating the evidence for or against a medical intervention. Systematic review with a hierarchy of evidence (RCTs at

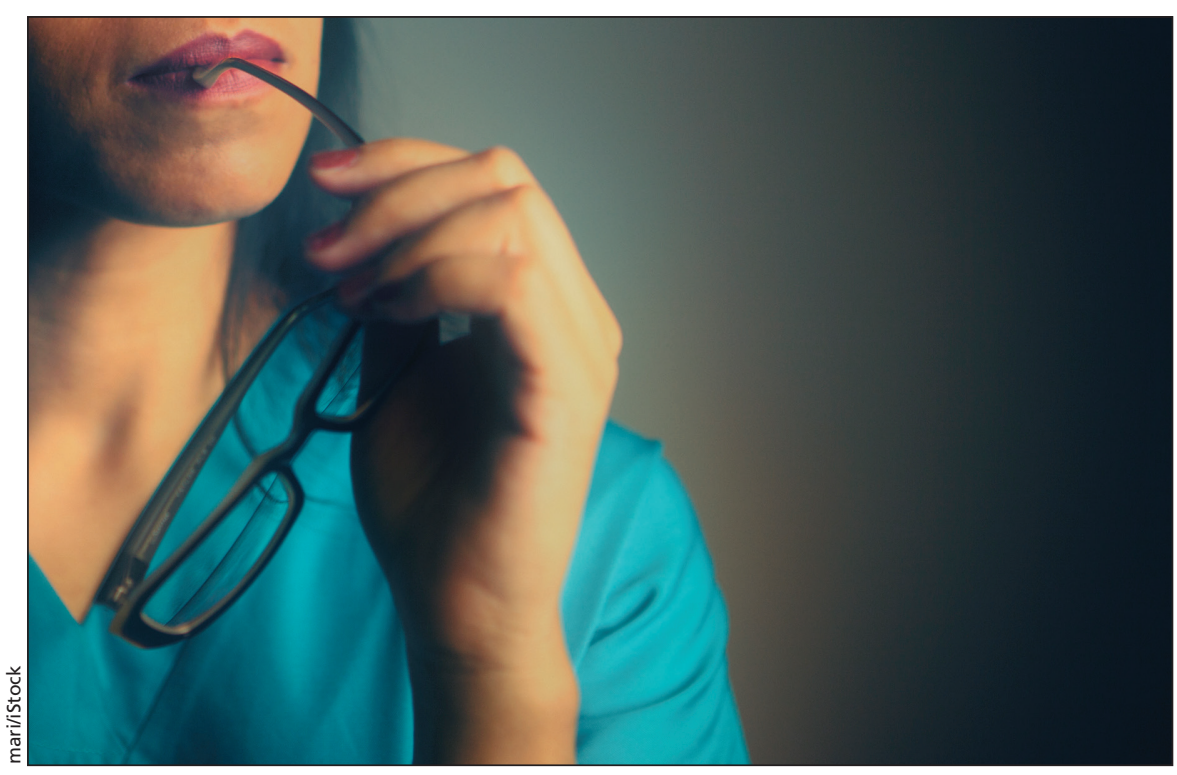

the top) and meta-analysis are standard techniques. The techniques come from epidemiology, and it would have been more descriptive to use the term epidemiologic medicine rather than evidencebased medicine. (On the other hand, the term evidence-based medicine has rhetorical power, because who would deny the importance of evidence?)

The need for translational medicine (first mentioned around 2002) ${ }^{4}$ became evident after a decade of emphasis on evidence-based medicine. The main focus of evidence-based medicine is phase III clinical trials. These trials are designed to determine the effectiveness of interventions in large populations. They take place after laboratory research, animal research, and phase I and II trials in humans (which focus on discovery, safety and establishing therapeutic doses). However, phase III trials do not take the place of the earlier research, and the earlier research is not properly judged by the standards of large-scale clinical trials. I claim that the bench-to-bedside meaning of translational medicine (i.e., the primary meaning of the term translational medicine) reflects an attempt to restore the balance between the various stages of research, and both fund and publish the earlier often more risky — stages of inquiry.
That is, translational medicine focuses on discovery and development of a new intervention, whereas evidence-based medicine focuses on testing and evaluating the new discovery. Furthermore, although evidence-based medicine offers explicit standards for experimental design and methods for aggregating the results of clinical trials, translational medicine offers little more than a metaphor (translation) to describe the difficult work of translating pure research into clinical applications. Perhaps the methods of translational medicine will improve, or perhaps we will need to accept that there is no logic of discovery and a metaphor is the best we can do.

Since the birth of scientific medicine in the late 19th century, the science of medicine has been contrasted with the art of medicine. The science has been variously described as including laboratory experiments, micromechanistic accounts (e.g., molecular mechanisms) and clinical trials. The art has typically encompassed a focus on care (rather than cure), an emphasis on the importance of the physician-patient relationship, and a concern with the ethical and humanistic side of medicine. Many people (both inside and outside the medical community) feel that the science side gets attention at the expense of the art side. Advances on the 
science side have often been followed by reactions from the art side.

Narrative medicine, which has roots in the bioethics literature of the 1980s, is the most prominent recent development in the medical humanities. Rita Charon's book, Narrative Medicine: Honoring the Stories of Illness, ${ }^{5}$ uses methods of literary analysis in primary care clinical settings, arguing that "good readers make good doctors." Narrative medicine is seen to fill the need for the art of medicine. It has been well-received in some clinical and academic settings and has also been discussed critically as being susceptible to the narrative fallacy (i.e., causal hypotheses are too readily constructed from sequences of events) and to the influence of constraining and politically dominant narratives. ${ }^{2,6}$

Evidence-based medicine has also been criticized for providing only statistical (epidemiologic) information about participants in clinical trials. or "cookbook" therapeutics of evidence-based medicine. At first, this sounds like an ethos similar to that of narrative medicine, but the word "personalized" is a bit of a misnomer because this meaning of personalized medicine has nothing to do with people (taken in the humanistic sense). Perhaps confusion was facilitated by the pervasive cultural equation of our personhood with our DNA. ${ }^{7}$ Not surprisingly, the term "personalized medicine" came to be used to refer to all kinds of personalization in medicine and, especially, to a caring and personal physician-patient relationship. This is confused and confusing. I am glad that it seems to have been replaced more recently with the term "precision medicine," which preserves the tailoring to individual cases without implying anything about bedside manner.

In his State of the Union address in January 2015, United States President Obama announced a \$215 million Preci-

\section{The ethos of personalized medicine is that it rejects the supposedly one-size-fits-all or cook-book therapeutics of evidence-based medicine.}

The best kind of evidence it can give is that patients with characteristics similar to the test population will have results statistically similar to the test population. It cannot predict the results for particular individuals. Moreover, when patients differ from the test population in medically significant ways, clinicians can only make educated guesses about what the results will be. It is not surprising that clinicians and patients want more precision and more certainty than the epidemiologic techniques of evidencebased medicine can offer.

Personalized medicine emerged in the early 2000s, with the goal of using genetic and other molecular information to make new discoveries and predict patient responses to treatments more accurately. Its ethos is that it rejects the supposedly one-size-fits-all sion Medicine Initiative. This has the explicit goal of moving beyond onesize-fits-all medicine to offer more specific treatments in subgroups of patients (e.g., those with shared somatic cell genetics or shared tumour cell genetics). This does not mean that the methods of evidence-based medicine can be bypassed. In fact, it means that - all other things being equal — we will need larger test populations to get statistically significant results about subpopulations. Precision medicine is accompanied by a hope that the interventions it produces will have more reliable and larger positive effects than the traditional, less precise interventions designed for broad diagnostic categories. When the size of the effect is larger and more reliable, test populations can be smaller and still reach statistical significance.

Precision medicine also offers a gen- eral strategy for translational research: look at the variability behind similar diagnoses and design interventions that utilize this variability. Although not precise enough to count as a logic of discovery, it is more specific than the metaphor of translation and a change from the usual strategy of designing interventions around similarities rather than differences.

A good example of successful precision medicine is the recent development of genomic treatments designed for cystic fibrosis, which target the misfolded protein underlying the disease. These misfolded proteins can be produced in a variety of ways because there are a number of possible mutations of the CFTR gene. New therapies have been tailored to specific mutations of CFTR and are showing promise (e.g., ivacaftor has been approved to treat patients with a G551D mutation).

Evidence-based medicine, translational medicine, narrative medicine and personalized/precision medicine emerged at specific times because of perceived lacunae in medical methodology and knowledge. In general, they supplement and sometimes modify, rather than replace, one another. Methods develop along with medical knowledge; they are not settled in advance.

\section{Miriam Solomon PhD}

Professor and Chair, Department of Philosophy, Temple University,

Philadelphia, $\mathrm{Pa}$.

\section{References}

1. Evidence-based medicine working group. Evidencebased medicine: a new approach to teaching the practice of medicine. JAMA 1992;268:2420-5.

2. Solomon M. Making medical knowledge. Oxford: Oxford University Press; 2015.

3. Baratz SR, Goodman C; Council on Health Care Technology. Improving consensus development for health technology assessment: an international perspective. Washington (DC): National Academy Press; 1990.

4. Pizzo P. Letter from the Dean. Stanford Medicine Magazine; Fall 2002. Available: http://sm.stanford. edu/archive/stanmed/2002fall/letter.html (accessed 2015 Nov. 8)

5. Charon R. Narrative medicine: honoring the stories of illness. Oxford; New York: Oxford University Press; 2006.

6. Segal J. Breast cancer narratives as public rhetoric: genre itself and the maintenance of ignorance. Ling Hum Sci 2007;3:3-23.

7. Nelkin D, Lindee MS. The DNA mystique: the gene as a cultural icon. New York: Freeman; 1995.

CMAJ 2016. DOI:10.1503/cmaj.150673 